\title{
Impact of sacubitril/valsartan on systolic heart failure: Right heart location and clustering analysis
}

\author{
Yanis Bouali ${ }^{1, A-D, F}$, Elena Galli ${ }^{1,2, E, F}$, Elise Paven ${ }^{1,2, E, F}$, Clement Laurini, ${ }^{1,2, B-D, F}$, \\ Hubert Arnaud ${ }^{1,2, B-D, F}$, Emmanuel Oger ${ }^{2, A, C, E, F}$, Erwan Donal ${ }^{2, A-F}$ \\ ${ }^{1}$ Department of Cardiology, Rennes University Hospital, LTSI, INSERM 1099, University of Rennes, France \\ ${ }^{2}$ EA Reperes, CHU Rennes, University of Rennes, France \\ A - research concept and design; $\mathrm{B}$ - collection and/or assembly of data; $\mathrm{C}$ - data analysis and interpretation; \\ $\mathrm{D}$ - writing the article; $\mathrm{E}$ - critical revision of the article; $\mathrm{F}$ - final approval of the article
}

Address for correspondence

Erwan Donal

E-mail: Erwan.DONAL@chu-rennes.fr

Funding sources

None declared

Conflict of interest

Prof. Erwan Donal received a research grant from Novartis France for this project. The other authors have no conflict of interest to declare.

Received on May 15, 2021

Reviewed on August 9, 2021

Accepted on 0ctober 28, 2021

Published online on December 17, 2021

Cite as

Bouali Y, Galli E, Paven E, et al. Impact of sacubitril/valsartan on systolic heart failure: Right heart location and clustering analysis. Adv Clin Exp Med. 2022;31(2):109-119. doi:10.17219/acem/143433

DOI

10.17219/acem/143433

Copyright

Copyright by Author(s)

This is an article distributed under the terms of the

Creative Commons Attribution 3.0 Unported (CC BY 3.0)

(https://creativecommons.org/licenses/by/3.0/)

\section{Abstract}

Background. Heart failure with reduced ejection fraction (HFrEF) is a heterogeneous syndrome. In heart failure (HF) classifications, right ventricle (RV) function was for a long time unrecognized in favor of left ventricular ejection fraction (LVEF). The response to sacubitril/valsartan might differ according to phenotypes and the impact of right ventricular characteristics on this response remains controversial.

Objectives. First, we applied clustering analysis in a HFrEF population undergoing sacubitril/valsartan treatment according to guidelines, to identify phenotypes and their associated clinical outcomes. Secondly, we evaluated RV-remodeling.

Materials and methods. It is a prospective, observational, single-center study conducted on 108 symptomatic patients (mean age $66 \pm 12.8$ years, $22.2 \%$ women). First, the clustering analysis was applied in a HFrEF population undergoing sacubitril/valsartan treatment, according to the guidelines, in order to identify phenotypes and clinical outcomes associated with them. Secondly, we evaluated RV-remodeling.

Results. Two distinct clusters were identified. Among the differences between phenotypes, RV (tricuspid annular plane systolic excursion (TAPSE) $16 \pm 4 \mathrm{~mm}$ compared to $19 \pm 4 \mathrm{~mm}, \mathrm{p}<0.001$; RV free wall strain $-19 \pm 5 \%$ compared to $-21 \pm 4 \%, p=0.046$; RV fraction area change (FAC) $31 \pm 9 \%$ compared to $38 \pm 9 \%, \mathrm{p}<0.001$ ), LV-filling pressure (E-wave deceleration time 138 (median: 41) ms compared to 180 (median: 94) ms, $\mathrm{p}<0.001$; E/e' 16.7 (median: 8.0) ms compared to 13.0 (median: 9.7) ms, $\mathrm{p}=0.02$ ) and creatinine level (106 $\pm 34 \mu \mathrm{mol} / \mathrm{L}$ compared to $90 \pm 19 \mu \mathrm{mol} / \mathrm{L}, p=0.002)$ were substantially different at the initiation of therapy. Major adverse cardiac events (MACEs) or death occurred in 38 out of 107 patients: $51.1 \%$ in cluster 1 compared to $24.2 \%$ in cluster $2(p=0.0074)$. A significant improvement in RV-functional parameters was observed under treatment. The TAPSE improved and correlated with the change in left ventricular (LV) function. Yet, it did not correlate with systolic pulmonary artery pressure (SPAP) and LV end-diastolic diameter.

Conclusions. The HFrEF phenotype characterized by more severe RV dysfunction has a worse prognosis during sacubitril/valsartan therapy. Both RV- and LV functions significantly improve when the patient is treated with sacubitril/valsartan.

Key words: remodeling, heart failure, clustering, right heart, sacubitril/valsartan 


\section{Background}

Chronic heart failure (CHF) is a complex and heterogeneous syndrome. ${ }^{1}$ Despite a good understanding of its physiopathology, the classifications used in clinical practice continue to rely on imperfect parameters, such as left ventricular ejection fraction (LVEF) ${ }^{2}$ or the New York Heart Association (NYHA) classification. ${ }^{3}$ However, the evidence in favor of a continuum of LV systolic failure exists. ${ }^{2}$ For instance, the DANISH trial perfectly demonstrated the limitations of LVEF in the stratification of arrhythmic risk and therefore the selection of patients for treatment. ${ }^{4}$ Right ventricle (RV) function remains absent in latest guidelines despite its pivotal place in the heart. Clustering analysis is an exploratory and hypothesis-generating approach that has been shown to play an important role in identifying subtypes of complex diseases. ${ }^{5}$ Using this approach, Ahmad et al. identified 4 phenotypically distinct and clinically meaningful CHF types responding differently to exercise programmes. ${ }^{6}$ These findings demonstrate the heterogeneity within heart failure with reduced ejection fraction (HFrEF) patients, and the existing need for the improved phenotyping, instead of focusing on few parameters, in order to enhance the therapeutic efficacy through a more personalized management. In their analysis, LVEF was the only echocardiographic parameter and was not statistically different among the clusters. ${ }^{6}$

Sacubitril/valsartan, a neprilysin inhibitor combined to an angiotensin II receptor antagonist, has been shown to improve HFrEF patient prognosis. ${ }^{7}$ This prognostic effect is associated with a striking improvement in LV-function and reverse remodeling. ${ }^{8}$ Despite these impressive results, the response in terms of prognosis is heterogeneous across baseline LVEF-spectrum. ${ }^{9}$ Unfortunately, RV failure was not included in PARADIGM-HF subgroup analysis, its prognosis value was not established and RV-remodeling remains poorly studied. ${ }^{10-13}$

\section{Objectives}

Therefore, first, we applied a clustering analysis in the HFrEF population undergoing sacubitril/valsartan treatment, according to the current recommendations, ${ }^{1}$ in order to identify phenotypes using clinical but also echocardiographic parameters at the inclusion. Then, we studied the clinical outcome across clusters using a composite primary endpoint including major adverse cardiac events and overall death. Secondly, we evaluated remodeling of the right heart.

\section{Materials and methods}

\section{Patients}

This was a prospective, observational, single-center study conducted from November 2015 to January 2018. A total of 108 symptomatic patients with HFrEF and an indication to receive sacubitril/valsartan according to current recommendations ${ }^{1}$ were prospectively and consecutively enrolled. They were followed in our heart failure (HF) program because they were severe and symptomatic. These were CHF patients without any acute event over the past 3 months and without any change in their treatment over the 6 weeks preceding the introduction of sacubitril/valsartan. The patients had more than 6 months of follow-up before their inclusion in the study. Clinical data, including age, sex, NYHA functional class, congestive signs, systolic and diastolic blood pressure (SBP and DBP), medical therapy, creatinine level and sinus rhythm, were assessed for each patient before the sacubitril/valsartan administration (baseline), and at the 12-month follow-ups. Coronary artery disease (CAD) was defined as a history of myocardial infarction (MI) or coronary revascularization or angiographic evidence of multiple-vessel disease or single-vessel disease with $\geq 75 \%$ stenosis of the left main or left proximal anterior descending artery. Transthoracic echocardiography was performed at baseline and at the 12-month follow-up. Vital status, hospitalization and ventricular arrhythmic events were extracted from hospital medical records or by interviewing the physicians in charge of the patients. The presence of ventricular arrhythmias was defined by the occurrence of sustained ventricular tachycardia demanding hospitalization or implantable cardioverter-defibrillator (ICD) appropriate shock. Major adverse cardiac events (MACEs) were defined as the composite of overall death, HF-related hospitalization and sustained ventricular arrhythmias. A previous report about this cohort has been published. ${ }^{14}$

The study was conducted in accordance with "Good Clinical Practice" Guidelines of the Declaration of Helsinki. All patients provided written informed consent for participation in the study. The study protocol was approved by the Comité de Protection des Personnes (CPP) Sud-Ouest et Outre Mer (CCP Sud Est V, No. ID RCB: 2017-A02217-46).

\section{Sacubitril/valsartan therapy}

All patients received the maximum tolerated HF treatment before the sacubitril/valsartan initiation. The $1^{\text {st }}$ dose of $24 / 26 \mathrm{mg}$ or $49 / 51 \mathrm{mg}$ was administered twice daily, according to the blood pressure, age and biological parameters. In patients switching from an angiotensin-converting enzyme (ACE) inhibitor, a washout period of $36 \mathrm{~h}$ was implemented before the initiation of the treatment. The uptitration of the treatment was performed at the discretion of the physicians up to a target dose of 97/103 mg twice daily, if well tolerated.

\section{Echocardiographic analysis}

Before the initiation of sacubitril/valsartan and at the 12-month follow-up, all patients underwent 
transthoracic 2D echocardiography using standard equipment (Vivid 9 or 95; GE Healthcare, Horten, Norway), supplied with a $3 \mathrm{~S}$ or M5S 3.5-mHz transducer, with the same imaging protocol. Bidimensional, colour Doppler, pulsed wave, and continuous wave Doppler data were stored on a dedicated work station for offline analysis (EchoPAC; GE Healthcare).

Cardiac dimensions and functions were measured according to the current recommendations..$^{15}$

The RV function was described using tricuspid annular plane systolic excursion (TAPSE), Doppler S velocity, RV free wall strain in apical RV-dedicated 4-chamber view, RV fraction area change (FAC) and Tei index (myocardial performance index (MPI)).

Diastolic function and LV filling pressure were quantified according to the recommendations. ${ }^{16}$ In patients with detectable tricuspid regurgitation, systolic pulmonary artery pressure (sPAP) was estimated. The RV-arterial coupling was estimated with the TAPSE/sPAP ratio. ${ }^{17}$

\section{Statistical analysis}

Data are presented as mean \pm standard deviation (SD), median (interquartile range (IQR)) and frequency/percentage depending on the nature of variables.

Prior to the analysis, missing data were imputed using the singular value decomposition (SVD) impute function within the impute package in $\mathrm{R}$ software (The R Project for Statistical Computing, Vienna, Austria). The percentage of missing values ranged from $0 \%$ to $50 \%$ (for TAPSE/ sPAP ratio).

Before the cluster analysis was performed, the Gower dissimilarity was used to measure the closeness between each observation. This measure implies standardization, which is set to range for interval and ordinal variables. A hierarchical cluster analysis was conducted in PROC CLUSTER (SAS v. 9.4; SAS Institute Inc., Cary, USA), using Ward's minimum variance method with standardization. All clustering was blinded to the clinical outcome data. To better define the relevant number of clusters, we looked for a consensus among 3 statistics - local peaks of the cubic clustering criterion (CCC) and pseudo-F statistic combined with a small value of the pseudo $\mathrm{t}^{2}$ statistic and a larger pseudo $t^{2}$ for the next cluster fusion. Once clusters were defined, they were compared in regard to demographic, clinical and echocardiographic characteristics.

For outcome analyses, Fisher's exact test (main outcome) or Kruskal-Wallis test (secondary outcomes) was used to test the independent associations between the clusters and the outcomes. Freedom from MACEs or death was plotted for both clusters using Kaplan-Meier curves, and between-cluster differences in freedom from events were tested using the log-rank test, Wilcoxon test and the -2 log likelihood-ratio (LR) test.

The comparison of changes from baseline to the 12 months follow-up was based on the signed-rank statistic test. For the null hypothesis, the mean change was equal to 0 .
Correlations between relative variation of TAPSE and relative variation of LVEF, global longitudinal strain (GLS), left ventricular end-diastolic diameter (LVEDD) and sPAP in overall population were estimated with Pearson's correlation coefficient.

\section{Results}

\section{Patient characteristics}

The characteristics of patients with missing values at baseline are depicted in Table 1.

The patients' mean age was $66 \pm 12.8$ years. Twenty-four (22.2\%) patients were female, and coronary artery disease (CAD) was observed in $52(48.1 \%)$ patients. Congestive signs at enrollment were present in 41 (39\%) patients. The mean creatinine level was $97 \pm 31 \mu \mathrm{mol} / \mathrm{L}$. The mean N-terminal prohormone of brain natriuretic peptide (NTproBNP) level was 1624 (IQR 897-2761) pg/mL. Seventyeight $(75 \%)$ patients were in sinus rhythm at enrollment.

\section{Echocardiographic parameters}

Echocardiographic characteristics and missing values of patients at baseline are depicted in Table 2 .

Our population had significantly reduced mean LVEF, mean cardiac index and LV dilatation at baseline. The median E/e' was in favor of elevated LV-filling pressures. The left atrium (LA) was dilated. The sPAP was elevated considering the mean tricuspid regurgitation peak velocity (TR Vmax). The RV function, characterized by mean TAPSE, free wall strain, Doppler S velocity, Tei index (MPI) and FAC, was slightly impaired. The right heart was dilated based on the mean tricuspid annulus diameter.

\section{Cluster analyses: patient and echocardiographic characteristics}

Cluster analysis identified 2 clusters. Patient characteristics across clusters are shown in Table 3, and echocardiographic characteristics are shown in Table 4.

Cluster 1 was smaller $(n=45)$ than cluster $2(n=63)$. In the $1^{\text {st }}$ cluster, patients had more comorbid conditions, with higher creatinine levels and higher rates of atrial fibrillation (AF). This cluster seemed to have more advanced HF pathology, with more patients in stage III or IV of the NYHA classification, the presence of congestive signs, and higher NT-proBNP levels. Regarding the treatment, $100 \%$ of patients used $\beta$ blockers. Loop diuretics were more often used in this cluster as aldosterone receptor antagonists.

Regarding echocardiographic parameters, cluster 1 included patients with lower LVEF $(27 \pm 11 \%$ compared to $30 \pm 8 \%, \mathrm{p}=0.045$ ), and LV diastolic function parameters 
Table 1. Baseline and 12-month follow-up population characteristics

\begin{tabular}{|c|c|c|c|c|}
\hline Characteristics & Values & $\begin{array}{c}\text { Baseline missing values } \\
\mathrm{n}(\%)\end{array}$ & $\begin{array}{l}\text { Baseline values* } \\
\mathrm{n}(\%)\end{array}$ & 12 -month FU values* $n(\%)$ \\
\hline Female, n (\%) & - & 0 & $24(22.2)$ & - \\
\hline Age [years] & - & 0 & $66.0 \pm 12.8$ & - \\
\hline BMI $\left[\mathrm{kg} / \mathrm{m}^{2}\right]$ & - & 0 & $27.8 \pm 5.10$ & - \\
\hline Creatinine $[\mu \mathrm{mol} / \mathrm{L}]$ & - & $25(23.1)$ & $97 \pm 31$ & $106 \pm 41$ \\
\hline $\mathrm{SBP}[\mathrm{mm} \mathrm{Hg}]$ & - & $18(16.7)$ & $122 \pm 19$ & $123 \pm 19$ \\
\hline \multirow{4}{*}{ NYHA class } & । & 0 & $4(3.70)$ & $31(39.7)$ \\
\hline & $\|$ & - & $78(72.2)$ & $38(48.7)$ \\
\hline & III & - & $21(19.4)$ & $9(11.5)$ \\
\hline & IV & - & $5(4.63)$ & $0(0)$ \\
\hline Congestive HF & - & $3(2.78)$ & $41(39.0)$ & $15(18.7)$ \\
\hline NT-proBNP [ng/L] & - & $27(25.0)$ & 1624 [897-2761] [min-max] & 1014 [412-2306] [min-max] \\
\hline \multirow{2}{*}{$C A D$} & $\mathrm{Ml}$ & 0 & $51(47.2)$ & - \\
\hline & $\mathrm{PCl}$ & - & $1(0.93)$ & - \\
\hline COPD & - & 0 & $16(14.8)$ & - \\
\hline Sinus rhythm & - & $4(3.70)$ & $78(75.0)$ & - \\
\hline$\beta$-blockers & - & $7(6.48)$ & $96(95.0)$ & $79(98.7)$ \\
\hline \multirow{4}{*}{ Loop diuretic } & no treatment & - & $20(21.3)$ & $23(30.3)$ \\
\hline & low dose ( $\leq 80 \mathrm{mg})$ & $14(13.0)$ & $57(60.6)$ & - \\
\hline & medium (120 mg) & - & $8(8.51)$ & - \\
\hline & high dose (>125 mg) & - & $9(9.57)$ & - \\
\hline $\begin{array}{l}\text { Aldosterone receptor } \\
\text { antagonists }\end{array}$ & - & $13(12.0)$ & $57(60.0)$ & $49(64.5)$ \\
\hline
\end{tabular}

BMI - body mass index; HF - heart failure; CAD - coronary artery disease; COPD - chronic obstructive pulmonary disease; NYHA - New York Heart Association; $\mathrm{SBP}$ - systolic blood pressure; NT-proBNP - N-terminal prohormone of brain natriuretic peptide; $\mathrm{Ml}$ - myocardial infarction; $\mathrm{PCl}$ - percutaneous coronary intervention; FU - follow-up; * mean \pm standard deviation (SD), median (Q1-Q3) or frequency (\%).

were more altered in regard to E-wave deceleration time (138 (median: 41) ms compared to 180 (median: 94) ms, p < 0.001), E/e' (16.7 (median: 8.0) ms compared to 13.0 (median: 9.7) $\mathrm{ms}, \mathrm{p}=0.022$ ) and left atrium volume in$\operatorname{dex}$ (LAVI) $\left(55 \pm 21 \mathrm{~mL} / \mathrm{m}^{2}\right.$ compared to $42 \pm 17 \mathrm{~mL} / \mathrm{m}^{2}$, $\mathrm{p}<0.001)$. The TR Vmax was higher $(3.10 \pm 0.44 \mathrm{~m} / \mathrm{s}$ compared to $2.92 \pm 0.45 \mathrm{~m} / \mathrm{s}, \mathrm{p}=0.037)$. The RV function was more impaired in terms of TAPSE, RV free wall strain and FAC (16 $\pm 4 \mathrm{~mm}$ compared to $19 \pm 4 \mathrm{~mm}, \mathrm{p}<0.001$; $-19 \pm 5 \%$ compared to $-21 \pm 4 \%$, p $=0.046 ; 31 \pm 9 \%$ compared to $38 \pm 9 \%, \mathrm{p}<0.001$, respectively). Right cavity remodeling was also significantly greater for the tricuspid annulus and right atrium (RA) $(40 \pm 7 \mathrm{~mm}$ compared to $37 \pm 7 \mathrm{~mm}, \mathrm{p}=0.010 ; 78$ (median: 45 ) $\mathrm{mL}$ compared to 51 (median: 36) $\mathrm{mL}, \mathrm{p}=0.001$, respectively). The TAPSE/sPAP ratio was more impaired $(0.37 \pm 0.15 \mathrm{~mm} / \mathrm{mm}$ Hg compared to $0.52 \pm 0.25 \mathrm{~mm} / \mathrm{mm} \mathrm{Hg}, \mathrm{p}=0.031)$.

\section{Follow-up}

The median clinical follow-up was 359 (median: 133) days. The MACEs or death occurred in 38 patients: $51.1 \%$ in cluster 1 as compared to $24.2 \%$ in cluster $2(\mathrm{p}=0.0074)$ (Fig. 1). The Kaplan-Meier survival curves (Fig. 1A) and stacked bar graphs (Fig. 1B) show that cluster 1 was at significantly higher risk for the primary endpoint of MACEs or overall death than cluster 2 .

\section{Right ventricular function improvement}

Functional parameters improvement was observed in the right heart, from baseline to 12-month follow-up, regarding TAPSE, TAPSE/sPAP ratio, RV free wall strain, and FAC (Fig. 2). The TAPSE, FAC and RV free wall strain were also significantly improved in each cluster.

Moreover, tricuspid annulus diameter was significantly decreased at 12-month follow-up ( $\mathrm{p}<0.001)$.

A significant LV reverse remodeling was observed from baseline to 12-month follow-up regarding LVEF ( $\mathrm{p}<0.0001)$, GLS ( $\mathrm{p}<0.0001)$ and LVEDD $(\mathrm{p}<0.0001)$. The LV diastolic function parameters, including E/A, E/e' and TR Vmax, were particularly improved from baseline to the 12-month follow-up. The LAVI did not significantly change (Fig. 3). 
Table 2. Baseline and 12-month follow-up echocardiographic characteristics

\begin{tabular}{|c|c|c|c|c|}
\hline Characteristics & $\begin{array}{c}\text { Baseline } \\
\text { missing values } \\
\mathrm{n}(\%)\end{array}$ & $\begin{array}{l}\text { Baseline } \\
\text { values* }\end{array}$ & $\begin{array}{l}\text { 12-month FU } \\
\text { values* }\end{array}$ & $\mathrm{p}$-value \\
\hline LV end-diastolic diameter [mm] & $2(1.85)$ & $64 \pm 9$ & $60 \pm 10$ & $<0.01$ \\
\hline LV end-systolic diameter [mm] & $6(5.55)$ & $54 \pm 11$ & $50 \pm 11$ & $<0.01$ \\
\hline LVEF (\%) & $2(1.85)$ & $29 \pm 9$ & $39 \pm 12$ & $<0.01$ \\
\hline Cardiac output [L/min] & $3(2.78)$ & $2.01 \pm 0.57$ & $2.36 \pm 0.74$ & $<0.01$ \\
\hline GLS (\%) & $22(20.4)$ & $-9(-11 ;-7)$ & $-13(-16 ;-9)$ & $<0.01$ \\
\hline Peak systolic dispersion [ms] & $22(20.4)$ & $67(57 ; 82)$ & $63(51 ; 74)$ & $<0.01$ \\
\hline$E / A$ & $26(24.1)$ & $1.3(0.8 ; 2.6)$ & $0.9(0.6 ; 1.5)$ & $<0.01$ \\
\hline DTE $[\mathrm{ms}]$ & $6(5.55)$ & $155(129 ; 195)$ & $190(148 ; 261)$ & $<0.01$ \\
\hline$E / e^{\prime}$ & $18(16.7)$ & $14(10 ; 21)$ & $11(9 ; 15)$ & $<0.01$ \\
\hline $\mathrm{LAVI}\left[\mathrm{mL} / \mathrm{m}^{2}\right]$ & $2(1.85)$ & $48 \pm 20$ & $47 \pm 20$ & 0.045 \\
\hline RA volume $\left[\mathrm{mL} / \mathrm{m}^{2}\right]$ & $6(5.55)$ & $62(41 ; 88)$ & $56(35 ; 73)$ & $<0.01$ \\
\hline TAPSE $[\mathrm{mm}]$ & $4(3.70)$ & $18 \pm 5$ & $21 \pm 5$ & $<0.01$ \\
\hline RV free wall strain (\%) & $28(25.9)$ & $-20 \pm 6$ & $-22 \pm 9$ & $<0.01$ \\
\hline $\mathrm{S}^{\prime}$ tricuspid $[\mathrm{cm} / \mathrm{s}]$ & $9(8.33)$ & $0.10 \pm 0.02$ & $0.11 \pm 0.02$ & 0.07 \\
\hline Tei-index TDI & $21(19.4)$ & $0.61 \pm 0.16$ & $0.53 \pm 0.13$ & $<0.01$ \\
\hline FAC (\%) & $15(13.9)$ & $35 \pm 10$ & $39 \pm 10$ & $<0.01$ \\
\hline Tricuspid annulus [mm] & $8(7.41)$ & $38 \pm 7$ & $33 \pm 8$ & $<0.01$ \\
\hline $\mathrm{TR} \vee \max [\mathrm{m} / \mathrm{s}]$ & $39(36.1)$ & $2.99 \pm 0.57$ & $2.72 \pm 0.61$ & $<0.01$ \\
\hline TAPSE/sPAP [mm/mm Hg] & $54(50)$ & $0.46 \pm 0.23$ & $0.62 \pm 0.31$ & $<0.01$ \\
\hline
\end{tabular}

DTE - E-wave deceleration time; FAC - fractional area change; GLS - global longitudinal strain; LAVI - left atrial volume index; LVEF - left ventricular ejection fraction; RA - right atrium; RV - right ventricle; sPAP - systolic pulmonary artery pressure; TAPSE - tricuspid annular plane systolic excursion; TDI - tissue doppler imaging; TR Vmax - tricuspid regurgitation peak velocity; FU - follow-up. * mean \pm standard deviation (SD), median (Q1-Q3).

Table 3. Baseline population characteristics across clusters

\begin{tabular}{|c|c|c|c|c|c|}
\hline Variables & Value & Total $1(n=45)$ & Cluster $(1 \mathrm{n}=45)$ & Cluster $2(n=63)$ & p-value \\
\hline Male sex & - & 84 & $84.4(38)$ & $73.0(46)$ & 0.159 \\
\hline Age [years] & - & - & $66.6 \pm 14.2$ & $65.6 \pm 11.9$ & 0.715 \\
\hline $\mathrm{BMI}\left[\mathrm{kg} / \mathrm{m}^{2}\right]$ & - & - & $28.6 \pm 5.42$ & $27.1 \pm 4.74$ & 0.134 \\
\hline Creatinine $[\mu \mathrm{mol} / \mathrm{L}]$ & - & - & $106 \pm 34$ & $90 \pm 19$ & 0.002 \\
\hline $\mathrm{SBP}[\mathrm{mm} \mathrm{Hg}]$ & - & - & $119 \pm 20$ & $123 \pm 14$ & 0.189 \\
\hline \multirow{2}{*}{ NYHA } & $|-| \mid$ & 82 & $53.3(24)$ & $92.1(58)$ & $<0.001$ \\
\hline & III-IV & 26 & $46.7(21)$ & $7.90(5)$ & - \\
\hline Congestive HF & - & 41 & $73.3(33)$ & $12.7(8)$ & $<0.001$ \\
\hline NT-proBNP & - & - & $2144(1894)$ & $1646(1510)$ & 0.003 \\
\hline CAD-MI & - & 51 & $42.2(19)$ & $50.8(32)$ & 0.379 \\
\hline COPD & 1 & 16 & $13.3(6)$ & $15.9(10)$ & 0.714 \\
\hline Sinus rhythm & 1 & 82 & $53.3(24)$ & $92.1(58)$ & $<0.001$ \\
\hline$\beta$-blockers & 1 & 103 & $100(45)$ & $92.1(58)$ & 0.054 \\
\hline Aldosterone receptor antagonists & 1 & 70 & $75.6(34)$ & $57.1(36)$ & 0.049 \\
\hline \multirow{3}{*}{ Loop diuretic } & no treatment & 20 & $4.44(2)$ & $28.6(18)$ & - \\
\hline & low dose ( $\leq 80$ mg) & 71 & $62.2(28)$ & $68.2(43)$ & $<0.001$ \\
\hline & high dose (>80 mg) & 17 & $33.3(15)$ & $3.17(2)$ & - \\
\hline
\end{tabular}

BMI - body mass index; HF - heart failure; CAD-MI - coronary artery disease-myocardial infarction; COPD - chronic obstructive pulmonary disease; NYHA - New York Heart Association; NT-proBNP - N-terminal prohormone of brain natriuretic peptide; SBP - systolic blood pressure. Data are presented as mean \pm standard deviation (SD) or median (interquartile range (IQR)). 
Table 4. Baseline echocardiographic characteristics across clusters

\begin{tabular}{|l|c|c|c|}
\hline \multicolumn{1}{|c|}{ Variable } & $\begin{array}{c}\text { Cluster 1 } \\
(\mathrm{n}=45)\end{array}$ & $\begin{array}{c}\text { Cluster } 2 \\
(\mathrm{n}=63)\end{array}$ & $p$-value \\
\hline LV end-diastolic diameter $[\mathrm{mm}]$ & $66 \pm 10$ & $62 \pm 8$ & 0.068 \\
\hline LVEF (\%) & $27 \pm 11$ & $30 \pm 8$ & 0.045 \\
\hline Cardiac output [mL/m²] & $1.91 \pm 0.55$ & $2.08 \pm 0.57$ & 0.138 \\
\hline GLS (\%) & $-9(2)$ & $-9(3)$ & 0.187 \\
\hline Peak systolic dispersion [ms] & $73.9(12.0)$ & $68.0(22.0)$ & 0.396 \\
\hline E/A & $2.21(0.33)$ & $1.27(1.44)$ & 0.627 \\
\hline DTE [ms] & $138(41)$ & $180(94)$ & $<0.001$ \\
\hline E/e' & $16.7(8.0)$ & $13.0(9.7)$ & 0.022 \\
\hline LAVI [mL/m²] & $55 \pm 21$ & $42 \pm 17$ & $<0.001$ \\
\hline RA volume [mL/m²] & $78(45)$ & $51(36)$ & $<0.001$ \\
\hline TAPSE [mm] & $16 \pm 4$ & $19 \pm 4$ & $<0.001$ \\
\hline Strain lat. RV (\%) & $-19 \pm 5$ & $-21 \pm 4$ & 0.046 \\
\hline Tei-index TDI & $0.62 \pm 0.15$ & $0.59 \pm 0.13$ & 0.245 \\
\hline FAC (\%) & $31 \pm 9$ & $38 \pm 9$ & $<0.001$ \\
\hline Tricuspid annulus [mm] & $40 \pm 7$ & $37 \pm 7$ & 0.010 \\
\hline TRVmax [m/s] & $3.10 \pm 0.44$ & $2.92 \pm 0.45$ & 0.037 \\
\hline TAPSE/sPAP [mm/mm Hg] & $0.37 \pm 0.15$ & $0.52 \pm 0.25$ & 0.031 \\
\hline
\end{tabular}

DTE - E-wave deceleration time; GLS - global longitudinal strain; $L A V I$ - left atrial volume index; LVEF - left ventricular ejection fraction; RA - right atrium; RV - right ventricle; sPAP - systolic pulmonary artery pressure; TAPSE - tricuspid annular plane systolic excursion; TDI - tissue doppler imaging; TR Vmax - tricuspid regurgitation peak velocity; FAC - fractional area change. Data are presented as mean \pm standard deviation (SD) or median (interquartile range (IQR)).

\section{Correlation of right ventricular function improvement}

Correlation between TAPSE improvement and LVEF, GLS, LV end-diastolic diameter and sPAP are presented in Fig. 4. The TAPSE improvement was significantly associated with LVEF and GLS improvement, whereas SPAP and LV end-diastolic diameter were not.

\section{Discussion}

The main findings of the study are as follows: 2 patient phenotypes were identified based on the study inclusion criteria (at the initiation time of sacubitril/valsartan), and the RV function seems to be a major factor distinguishing these subsets. Cluster 1 included significantly more symptomatic and congestive patients with higher NTproBNP levels, where RV-functional parameters were more impaired concomitantly to LVEF, LV diastolic function and renal function abnormalities. Patients included in cluster 1 had a worse prognosis regarding the primary outcome of MACEs or death. The RV function under sacubitril/valsartan was significantly improved, and TAPSE improvement significantly correlated with change in LVEF and GLS, but not with SPAP and LVEDD.
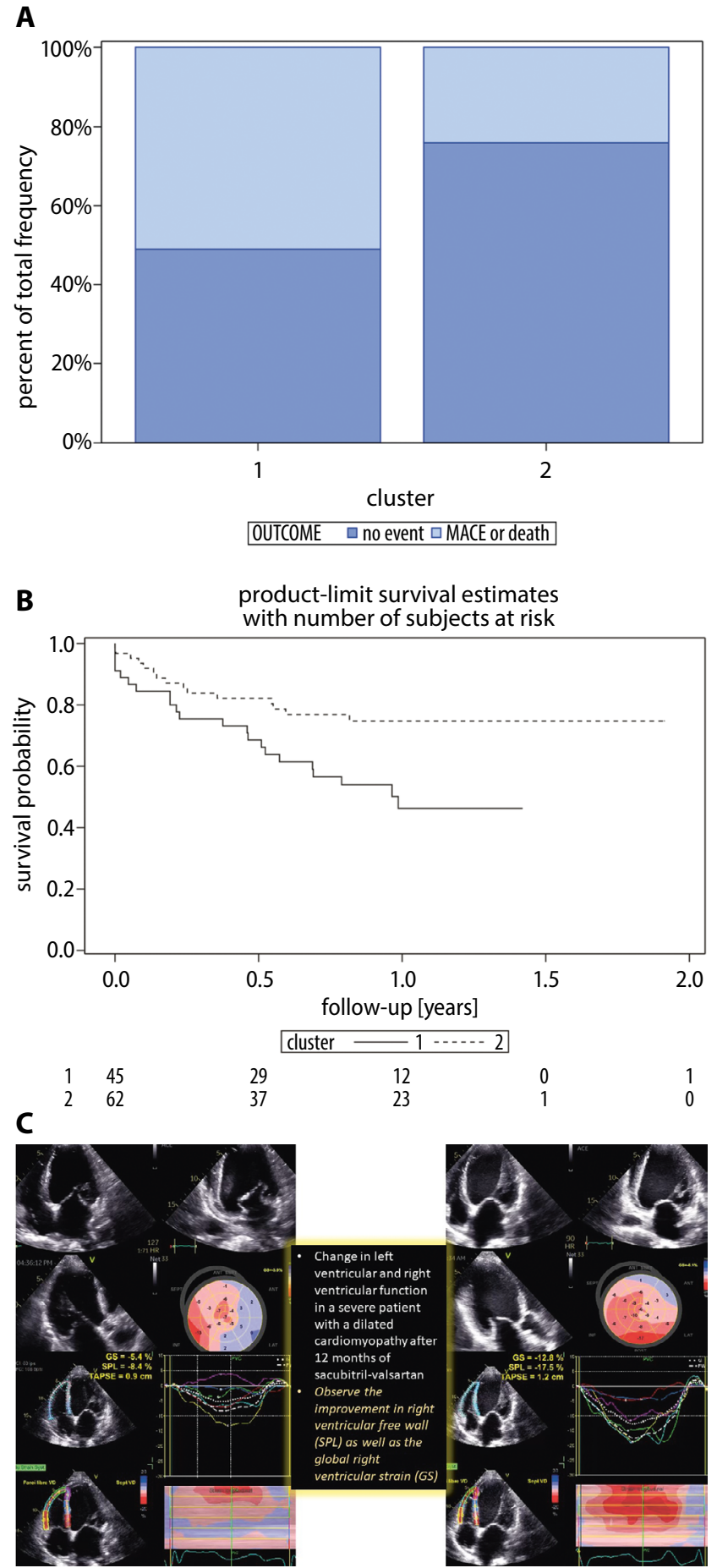

Fig. 1. A. Stacked bar graph of outcome by cluster; B. Kaplan-Meier survival curve for time to major adverse cardiac event (MACE) or overall death. Thirty-eight MACEs or overall deaths occurred among 107 patients (1 lost to follow-up); 51.1\% (23 MACEs or deaths out of 45 patients) compared to $24.2 \%$ (15 MACEs or deaths out of 62 patients), $p=0.0074$; C. The example of left ventricular (LV) and right ventricular function at inclusion and 1 year later

\section{Prognosis role of baseline RV failure in HFrEF using clustering analysis}

The cluster analysis allowed for the identification of 2 distinct meaningful demographic, clinical and echocardiographic phenotypes in a population of patients with 

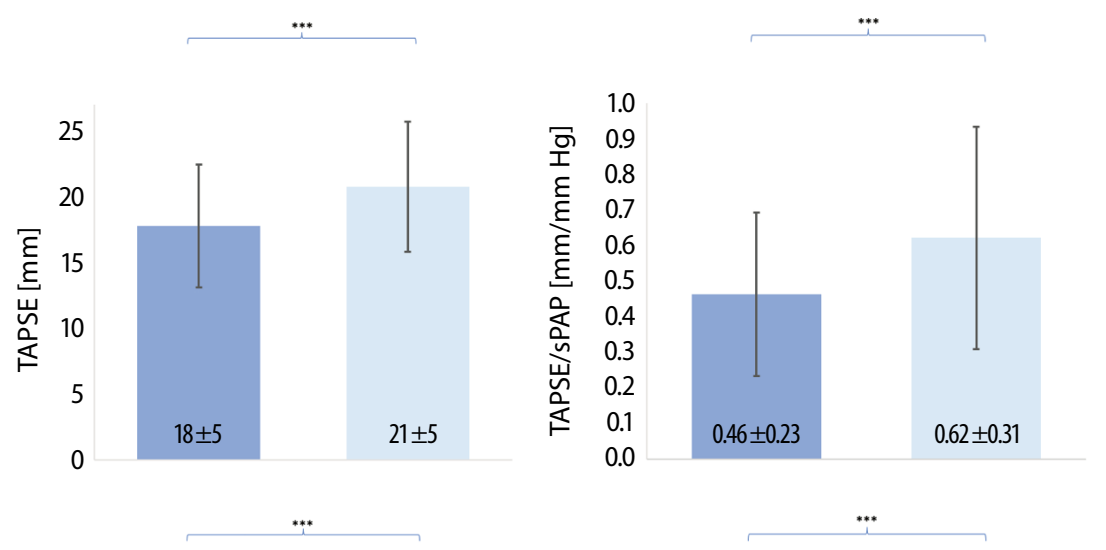

Fig. 2. Evolution of right ventricle (RV) function parameters under sacubitril/valsartan in overall population. Tricuspid annular plane systolic excursion (TAPSE), RV fraction area change (FAC) and RV free wall strain significantly improved in each cluster $(p<0.05)$. Mean \pm standard deviation (SD)

${ }^{*} p<0.05$; ${ }^{*} p<0.01$; *** $p<0.001$; sPAP - systolic pulmonary artery pressure.
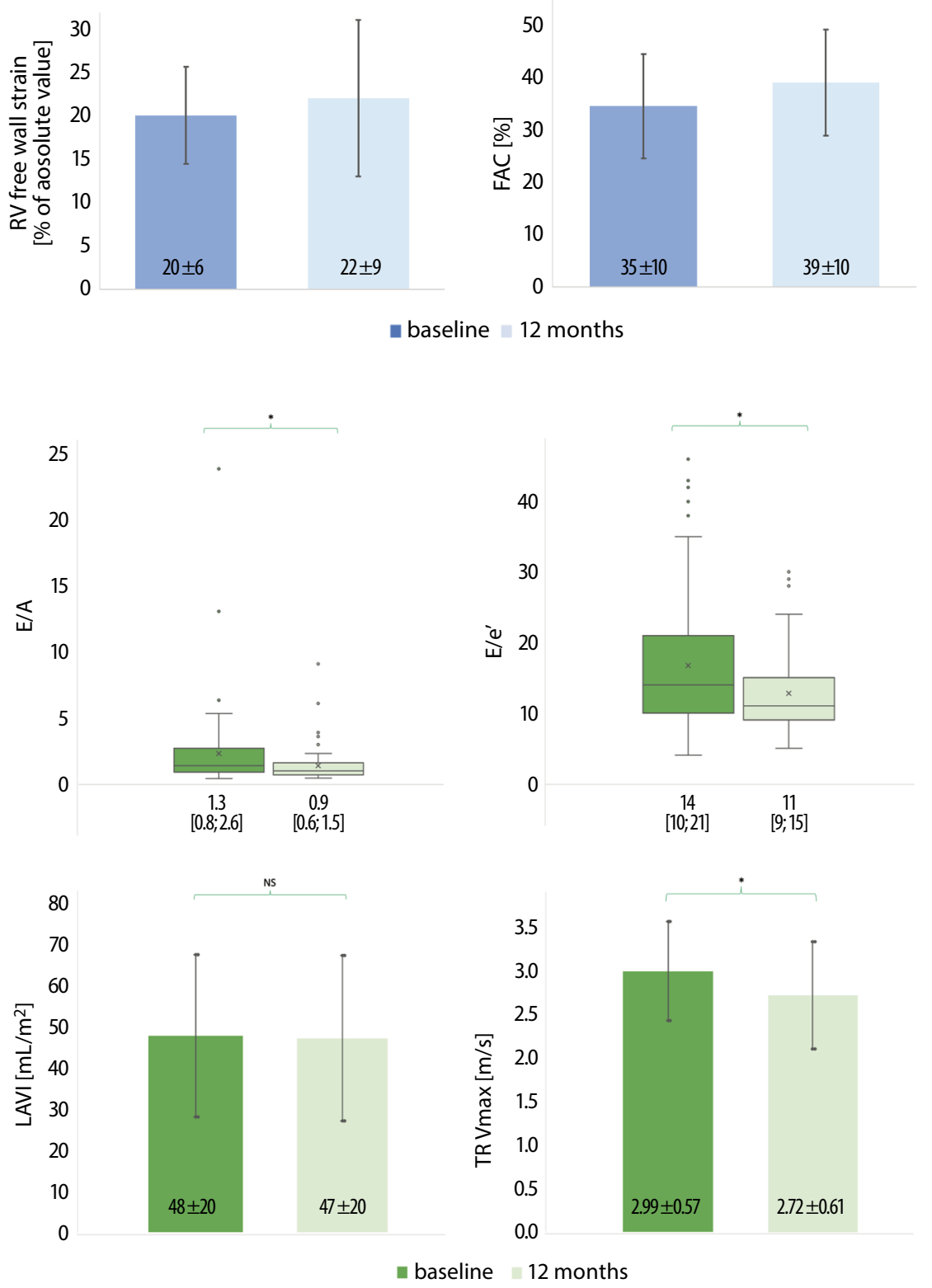

Fig. 3. Evolution of left ventricular (LV) diastolic function parameters under sacubitril/valsartan in the whole study population. Mean \pm standard deviation (SD) or median (Q1-Q3)

${ }^{*} p<0.05 ;{ }^{* *} p<0.01 ;{ }^{* * *} p<0.001 ;$ LAVI - left atrial volume index; TR Vmax - tricuspid regurgitation peak velocity.

chronic HFrEF receiving sacubitril/valsartan treatment. All clustering was blinded to the clinical outcome data. Yet, we demonstrate that cluster 1, representing a more advanced stage of HFrEF, has a significant dismal prognosis.
Our results seem to be in accordance with existing data. In the ESCAPE trial, renal dysfunction and NT-proBNP level, as well as HF-ACTION population were associated with a higher risk of clinical outcome in a cluster 

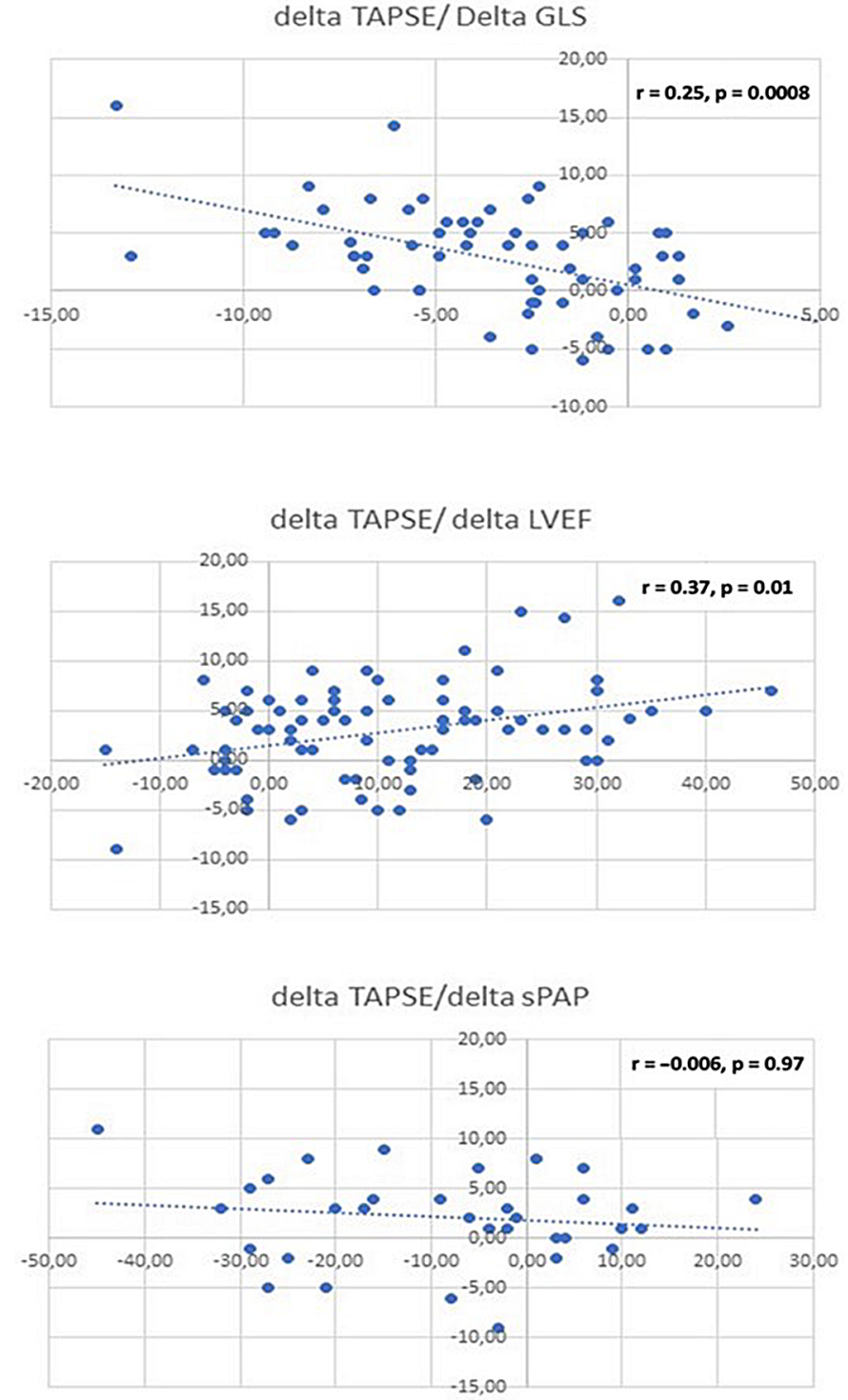

delta TAPSE/ delta LVEDD

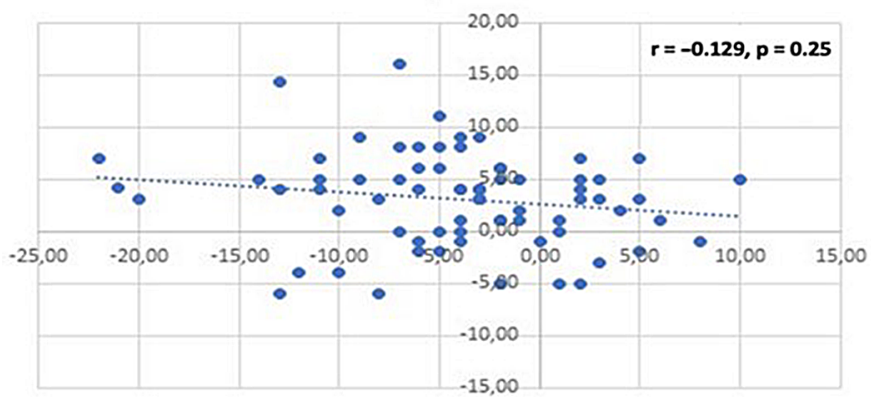

Fig. 4. Correlation between tricuspid annular plane systolic excursion (TAPSE) relative variation and left ventricular ejection fraction (LVEF), global longitudinal strain (GLS), systolic pulmonary artery pressure (sPAP), and left ventricular end-diastolic diameter (LVEDD) relative variation at 12-month follow-up in overall population

consisting mostly of older patients with ischemic cardiomyopathy, AF and diuretics. ${ }^{17}$ In a Swedish population of HF patients, the cluster that had the worst prognosis consisted of patients with the most advanced age, more important adverse symptoms, lowest creatinine clearance, and the highest NT-proBNP levels despite diuretic use, AF and MI rate (24\% of patients with HF had preserved ejection fraction (EF) in this registry). ${ }^{18}$ Curiously, no differences were observed in terms of age or frequency of ischemic etiology in our cohort.

Cluster 1, exhibiting a more severe clinical and echocardiographic profile with documented overload and activation of neurohormones (which are one of the main therapeutic targets of sacubitril/valsartan ${ }^{7}$ ), had the most unfavorable prognosis. This result might seem obvious but was never demonstrated until now. Thus, it could have an important clinical impact. Indeed, sacubitril/valsartan has not been first introduced in this study. It has been used in patients already uptitrated with ACE-inhibitors, like in the PARADIGM-HF trial. ${ }^{7}$ Nevertheless, our results encourage a much earlier therapy introduction, especially in the youngest patients. The main risk of this therapy in patient expected to have a dismal prognosis would be wasting time and removing the patient from a group with indication for transplant. Heterogenous response across LVEF spectrum was observed. ${ }^{9}$ Sacubitril/valsartan is superior to enalapril. Neverthelesss, the examination of the LV only cannot help in predicting the response expected at the introduction of the therapy. ${ }^{1}$ Our study has the advantage of providing other parameters, such as RV function parameters, which were not included in cluster analysis of HF ACTION, ESCAPE and the Swedish registry, despite the prognosis burden of the RV failure. ${ }^{19}$ Indeed, a major result of our study is a significant baseline RV failure regarding TAPSE, FAC and RV-free wall strain in cluster 1 , as compared to cluster 2 . Considering the TAPSE/ sPAP ratio, RV pulmonary arterial coupling seemed to be impaired in cluster 1 , indicating a lack of RV-adaptation to an increased afterload, and therefore an altered contractile reserve. ${ }^{16}$ Bosch et al. demonstrated the independent prognostic value of abnormal RV-pulmonary arterial coupling in such patients. ${ }^{19}$

Given the significant improvement of each parameter in both clusters, baseline RV failure seems to be involved in the prognosis more than RV function improvement. The patients from cluster 1 might have been switched to sacubitril/valsartan too late (once RV failure was too advanced). Prospective randomized trials are necessary to compare the introduction of sacubitril/valsartan before and after RV dysfunction related to the occurrence of HFrEF. For the most severe cases, the switch to sacubitril/valsartan could be proposed without delaying the decision-making process for more invasive therapeutic strategies, such as ventricular assist devices or transplantation.

\section{$\mathrm{RV}$ failure within the pathological process of HFrEF}

Cluster 1 requires higher doses of loop diuretics and mineralocorticoid receptor antagonists in patients 
suffering from a cardiorenal syndrome (type II). ${ }^{20}$ Despite an important prevalence of renal insufficiency in HF patients, the physiopathology of this syndrome remains largely open to discussion. In the context of HF, the association of renal impairment, even moderate, with poor prognosis is well known. ${ }^{21}$ The NT-proBNP, a major prognostic factor in $\mathrm{HF}^{22}$ is known to be higher in cases of renal failure, underlying the coherence of the results we observed with our cohort. The association between higher creatinine and NT-proBNP levels and RV dysfunction (and right cavities remodeling), highlighted by our findings, is consistent with existing data. ${ }^{20}$ The RV function, more than LV function, seems to have a major influence on cardiorenal syndrome with the elevated central venous pressures leading to renal venous hypertension and to the reduction of intrarenal blood flow. ${ }^{23}$ The independence of the association between elevated central venous pressures and renal dysfunction was demonstrated, and right atrial pressure was shown to be a predictor of allcause mortality. ${ }^{24}$ Although RV failure was never directly correlated to renal failure, our findings suggest a possible $\mathrm{RV}$ role in the genesis of elevated central venous pressures and in type II cardiorenal syndrome. The cause-effect relationship remains to be elucidated, and further studies are needed.

The concomitant presence of atrial arrhythmias and RV failure in cluster 1 is consistent. In our analysis, $92 \%$ of patients in cluster 2 were in sinus rhythm, compared with only $53 \%$ in cluster 1 , in accordance with the difference in right atrial and LA volumes between clusters. Indeed, atrial arrhythmias were identified as an independent prognostic factor of RV dysfunction in left-sided HF patients. ${ }^{19}$ The atrial arrhythmias were also largely described as a major prognostic factor in HFrEF. ${ }^{25}$

\section{Improvement of RV-functional parameters with sacubitril/valsartan}

One of the main results of this study was the capability of the right heart to improve with sacubitril/ valsartan. This is important as RV recovery in HFrEF patients is associated with an improved prognosis. ${ }^{26}$ According to the previous studies, although it is demonstrated and observed in practice that sacubitril/valsartan decreases pulmonary pressures, Correale et al. ${ }^{12}$ were the only researchers to have recently prospectively shown the associated improvement in RV function in regard to TAPSE. ${ }^{10,11,13,27}$ Yenerçağ et al. ${ }^{28}$ retrospectively showed the significant increase of TAPSE, FAC and MPI at 6-month follow-up. Supposedly, studies that did not show any significant RV improvement did not have follow-up long enough. Our cohort has the advantage to be the first to allow for a multiparametric assessment of RV function. It brings strength to the results despite the limited size of the cohort. Several parameters that could be required for the best assessment of RV function and its change over time were used. ${ }^{14,29}$ Tricuspid annulus diameter was used as a surrogate marker of RV remodeling. It significantly decreased in size over time, confirming the right heart reverse remodeling that could happen over time, especially in cluster 2 .

\section{Correlations of RV function improvement under sacubitril/valsartan}

In this study, it was demonstrated that switching from an ACE inhibitor to sacubitril/valsartan positively impacts loading conditions and diastolic filling patterns, as well as TASPE/sPAP ratio and RV function parameters. Therefore, the RV afterload decrease could be a possible mechanism of RV function improvement. However, TAPSE improvement was not significantly correlated with sPAP but with LVEF and GLS. It confirms the key role of systolic RV-LV interactions involved in the pathological process of HFrEF. ${ }^{19}$ This correlation was already described under optimal medical treatment. The RV FAC normalization was significantly associated with subsequent LVreverse remodeling, but only at 24 months, and sacubitril/ valsartan was not introduced in this study. ${ }^{30} \mathrm{~A}$ recent publication suggested a possible association between LVand RV-functional reverse remodeling, but no correlation analyses were performed. ${ }^{31}$ In the field of cardiac resynchronization, a post hoc analysis of MADIT-CRT trial demonstrated that significant RV function improvement was correlated with change in LVEF and LV end-diastolic volume but not in SPAP. ${ }^{32}$ The LV contribution to RV ejection was previously described ${ }^{33}$ and this interdependence is anatomically supported by shared myocardial fibers through the septum or free wall epicardial layer. According to some authors, RV longitudinal deformation holds the major role in RV ejection, ${ }^{34,35}$ despite the scarcity of RV longitudinal fibers. The interventricular septum shortening might play a crucial role in the interaction between right and left ventricles. In dilated cardiomyopathy, LV spherization leads to an alteration of septal fibres' helical orientation, turning to a more transversal pattern, and decreasing RV longitudinal deformation mediated by the septum. ${ }^{34}$ It results in lower mechanical efficiency and afterload adaptation, responsible for RV dysfunction followed by dilation. Consequently, TR worsens the vicious circle of HF. Therefore, we can formulate a hypothesis that LV reverse remodeling, already demonstrated under sacubitril/valsartan, ${ }^{8}$ corrects fibres' orientation and their contribution to longitudinal RV systolic function. In our study, the importance of longitudinal deformation in RV ejection could explain the stronger correlation of TAPSE improvement with GLS, rather than LVEF. The downregulation of fibrosis signaling mediated by sacubitril/valsartan ${ }^{36}$ could be involved, enhancing LV fibers contractility and their contribution to RV ejection. The direct effect of sacubitril/valsartan on RV cannot be ruled out. 


\section{Limitations}

Several limitations of this study must be considered. First, the cohort was prospective, but limited in size and monocentric. Second, it is challenging to extrapolate the prognostic impact of the severity of the disease and the impact of sacubitril/valsartan introduction. It could have been interesting to have a group of patients in which sacubitril/ valsartan would have been introduced before, as recommended by the American guidelines, ${ }^{37}$ in order to make a comparison in terms of a prognosis impact possible.

\section{Conclusion}

According to the clustering analysis, 2 phenotypes of HFrEF patients were generated, where RV failure, apart from AF, LV dysfunction and renal dysfunction at the initiation of therapy appears to be an important prognostic determinant during sacubitril/valsartan therapy. The RV function, similarly to LV function, significantly improves under sacubitril/valsartan, as indicated by TAPSE, FAC and RV free wall strain.

\section{ORCID iDs}

Yanis Bouali (10) https://orcid.org/0000-0001-9923-6547 Elena Galli 1 [ https://orcid.org/0000-0001-6692-9101 Elise Paven (1) https://orcid.org/0000-0003-4543-2141 Clement Laurin (1) https://orcid.org/0000-0002-1697-2504 Hubert Arnaud (10 https://orcid.org/0000-0002-4994-1165 Emmanuel Oger (1) https://orcid.org/0000-0001-9837-2977 Erwan Donal (10) https://orcid.org/0000-0003-2677-3389

\section{References}

1. Ponikowski P, Voors AA, Anker SD, et al. 2016 ESC Guidelines for the diagnosis and treatment of acute and chronic heart failure: The Task Force for the diagnosis and treatment of acute and chronic heart failure of the European Society of Cardiology (ESC) developed with the special contribution of the Heart Failure Association (HFA) of the ESC. Eur Heart J. 2016;37(27):2129-2200. doi:10.1093/eurheartj/ehw128

2. Triposkiadis F, Butler J, Abboud FM, et al. The continuous heart failure spectrum: Moving beyond an ejection fraction classification. Eur Heart J. 2019;40(26):2155-2163. doi:10.1093/eurheartj/ehz158

3. Caraballo C, Desai NR, Mulder $\mathrm{H}$, et al. Clinical implications of the $\mathrm{New}$ York Heart Association classification. J Am Heart Assoc. 2019;8(23): e014240. doi:10.1161/JAHA.119.014240

4. Køber L, Thune JJ, Nielsen JC, et al. Defibrillator implantation in patients with nonischemic systolic heart failure. N Engl J Med. 2016;375(13): 1221-1230. doi:10.1056/NEJMoa1608029

5. McLachlan GJ. Cluster analysis and related techniques in medical research. Stat Methods Med Res. 1992;1(1):27-48. doi:10.1177/096228 029200100103

6. Ahmad T, Pencina MJ, Schulte PJ, et al. Clinical implications of chronic heart failure phenotypes defined by cluster analysis. J Am Coll Cardiol. 2014;64(17):1765-1774. doi:10.1016/j.jacc.2014.07.979

7. McMurray JJV, Packer M, Desai AS, et al. Angiotensin-neprilysin Inhibition versus enalapril in heart failure. NEnglJMed. 2014;371(11):993-1004. doi:10.1056/NEJMoa1409077

8. Januzzi JL, Prescott MF, Butler J, et al. Association of change in N-terminal pro-B-type natriuretic peptide following initiation of sacubitrilvalsartan treatment with cardiac structure and function in patients with heart failure with reduced ejection fraction. JAMA. 2019;322(11): 1085-1095. doi:10.1001/jama.2019.12821
9. Solomon SD, Claggett B, Desai AS, et al. Influence of ejection fraction on outcomes and efficacy of sacubitril/valsartan (LCZ696) in heart failure with reduced ejection fraction: The prospective comparison of ARNI with ACEl to determine impact on global mortality and morbidity in heart failure (PARADIGM-HF) trial. Circ Heart Fail. 2016;9(3): e002744. doi:10.1161/CIRCHEARTFAILURE.115.002744

10. Bayard G, Da Costa A, Pierrard R, Roméyer-Bouchard C, Guichard JB, Isaaz K. Impact of sacubitril/valsartan on echo parameters in heart failure patients with reduced ejection fraction: A prospective evaluation. Int J Cardiol Heart Vasc. 2019;25:100418. doi:10.1016/j.ijcha.2019. 100418

11. Romano $G$, Vitale $G$, Ajello $L$, et al. The effects of sacubitril/valsartan on clinical, biochemical and echocardiographic parameters in patients with heart failure with reduced ejection fraction: The "hemodynamic recovery". J Clin Med. 2019;8(12):2165. doi:10.3390/jcm8122165

12. Correale M, Mallardi A, Mazzeo P, et al. Sacubitril/valsartan improves right ventricular function in a real-life population of patients with chronic heart failure: The Daunia Heart Failure Registry. Int J Cardiol Heart Vasc. 2020;27:100486. doi:10.1016/j.ijcha.2020.100486

13. Polito MV, Silverio A, Rispoli A, et al. Clinical and echocardiographic benefit of sacubitril/valsartan in a real-world population with HF with reduced ejection fraction. Sci Rep. 2020;10(1):6665. doi:10.1038/ s41598-020-63801-2

14. Lang RM, Badano LP, Mor-Avi V, et al. Recommendations for cardiac chamber quantification by echocardiography in adults: An update from the American Society of Echocardiography and the European Association of Cardiovascular Imaging. Eur Heart J Cardiovasc Imaging. 2015;16(3):233-270. doi:10.1093/ehjci/jev014

15. Nagueh SF, Smiseth OA, Appleton CP, et al. Recommendations for the evaluation of left ventricular diastolic function by echocardiography: An update from the American Society of Echocardiography and the European Association of Cardiovascular Imaging. J Am Soc Echocardiogr. 2016;29(4):277-314. doi:10.1016/j.echo.2016.01.011

16. Guazzi M, Bandera F, Pelissero G, et al. Tricuspid annular plane systolic excursion and pulmonary arterial systolic pressure relationship in heart failure: $\mathrm{An}$ index of right ventricular contractile function and prognosis. Am J Physiol Heart Circ Physiol. 2013;305(9):H1373-H1381. doi:10.1152/ajpheart.00157.2013

17. Ahmad T, Desai N, Wilson F, et al. Clinical implications of cluster analysis-based classification of acute decompensated heart failure and correlation with bedside hemodynamic profiles. PLOSONE. 2016;11(2): e0145881. doi:10.1371/journal.pone.0145881

18. Ahmad T, Lund LH, Rao $P$, et al. Machine learning methods improve prognostication, identify clinically distinct phenotypes, and detect heterogeneity in response to therapy in a large cohort of heart failure patients. J Am Heart Assoc. 2018;7(8):e008081. doi:10.1161/JAHA. 117.008081

19. Bosch L, Lam CSP, Gong L, et al. Right ventricular dysfunction in leftsided heart failure with preserved versus reduced ejection fraction. Eur J Heart Fail. 2017;19(12):1664-1671. doi:10.1002/ejhf.873

20. Rangaswami J, Bhalla V, Blair JEA, et al. Cardiorenal syndrome: Classification, pathophysiology, diagnosis, and treatment strategies. A scientific statement from the American Heart Association. Circulation. 2019;139(16):e840-e878. doi:10.1161/CIR.0000000000000664

21. Damman K, Valente MAE, Voors AA, O'Connor CM, van Veldhuisen DJ, Hillege HL. Renal impairment, worsening renal function, and outcome in patients with heart failure: An updated meta-analysis. Eur Heart J. 2014;35(7):455-469. doi:10.1093/eurheartj/eht386

22. Salah K, Stienen S, Pinto YM, et al. Prognosis and NT-proBNP in heart failure patients with preserved versus reduced ejection fraction. Heart. 2019;105(15):1182-1189. doi:10.1136/heartjnl-2018-314173

23. Mullens W, Abrahams Z, Francis GS, et al. Importance of venous congestion for worsening of renal function in advanced decompensated heart failure. J Am Coll Cardiol. 2009;53:589-596. doi:10.1016/j.jacc. 2008.05.068

24. Damman K, van Deursen VM, Navis G, Voors AA, van Veldhuisen DJ, Hillege HL. Increased central venous pressure is associated with impaired renal function and mortality in a broad spectrum of patients with cardiovascular disease. J Am Coll Cardiol. 2009;53(7):582-588. doi:10.1016/j.jacc.2008.08.080 
25. Olsson LG, Swedberg K, Ducharme A, et al. Atrial fibrillation and risk of clinical events in chronic heart failure with and without left ventricular systolic dysfunction: Results from the Candesartan in Heart failureAssessment of Reduction in Mortality and Morbidity (CHARM) Program. J Am Coll Cardiol. 2006;47(10):1997-2004. doi:10.1016/j.jacc.2006.01.060

26. Dini FL, Carluccio E, Simioniuc A, et al. Right ventricular recovery during follow-up is associated with improved survival in patients with chronic heart failure with reduced ejection fraction. Eur J Heart Fail. 2016;18(12):1462-1471. doi:10.1002/ejhf.639

27. Zern EK, Cheng S, Wolfson AM, et al. Angiotensin receptor-neprilysin inhibitor therapy reverses pulmonary hypertension in endstage heart failure patients awaiting transplantation. Circ Heart Fail. 2020;13(2):e006696. doi:10.1161/CIRCHEARTFAILURE.119.006696

28. Yenerçağ $M$, Arslan U, Dereli S, Çoksevim M, Doğduş M, Kaya A Effects of angiotensin receptor neprilysin inhibition on pulmonary arterial stiffness in heart failure with reduced ejection fraction Int J Cardiovasc Imaging. 2021;37(1):165-173. doi:10.1007/s10554-02001973-8

29. Carluccio E, Biagioli P, Alunni G, et al. Prognostic value of right ventricular dysfunction in heart failure with reduced ejection fraction: Superiority of longitudinal strain over tricuspid annular plane systolic excursion. Circ Cardiovasc Imaging. 2018;11(1):e006894. doi:10.1161/ CIRCIMAGING.117.006894

30. Merlo M, Gobbo M, Stolfo D, et al. The prognostic impact of the evolution of RV function in idiopathic DCM. JACC Cardiovasc Imaging. 2016;9(9):1034-1042. doi:10.1016/j.jcmg.2016.01.027
31. Santiago-Vacas E, Lupón J, Gavidia-Bovadilla G, et al. Pulmonary hypertension and right ventricular dysfunction in heart failure: Prognosis and 15-year prospective longitudinal trajectories in survivors. Eur J Heart Fail. 2020;22(7):1214-1225. doi:10.1002/ejhf.1862

32. Campbell $P$, Takeuchi $M$, Bourgoun $M$, et al. Right ventricular function, pulmonary pressure estimation, and clinical outcomes in cardiac resynchronization therapy. Circ Heart Fail. 2013;6(3):435-442. doi:10.1161/CIRCHEARTFAILURE.112.000127

33. Santamore WP, Lynch PR, Heckman JL, Bove AA, Meier GD. Left ventricular effects on right ventricular developed pressure. J Appl Physiol. 1976;41(6):925-930. doi:10.1152/jappl.1976.41.6.925

34. Saleh S, Liakopoulos OJ, Buckberg GD. The septal motor of biventricular function. Eur J Cardiothorac Surg. 2006;29(Suppl 1):S126-S138. doi:10.1016/j.ejcts.2006.02.048

35. Buckberg G, Hoffman JIE. Right ventricular architecture responsible for mechanical performance: Unifying role of ventricular septum. J Thorac Cardiovasc Surg. 2014;148(6):3166-3171.e4. doi:10.1016/ j.jtcvs.2014.05.044

36. Zile MR, O'Meara E, Claggett B, et al. Effects of sacubitril/valsartan on biomarkers of extracellular matrix regulation in patients with $\mathrm{HFrEF}$. J Am Coll Cardiol. 2019;73(7):795-806. doi:10.1016/j.jacc.2018.11.042

37. Yancy CW, Jessup M, Bozkurt B, et al. 2017 ACC/AHA/HFSA Focused Update of the 2013 ACCF/AHA Guideline for the Management of Heart Failure: A Report of the American College of Cardiology/American Heart Association Task Force on Clinical Practice Guidelines and the Heart Failure Society of America. J Am Coll Cardiol. 2017; 70(6):776-803. doi:10.1016/j.jacc.2017.04.025 